\title{
Exploring the Learning Mechanism in Educational Games
}

\author{
Kristian Kiili and Harri Ketamo \\ Tampere University of Technology, Pori, Finland
}

\begin{abstract}
The main aim of this paper is to evaluate the problembased gaming model that tries to explain the learning process in educational games. The model was studied through Geometry game aimed for pre-school children $(N=24)$. The game relays on learning by teaching approach and involves AI-engine modeling the human concept learning structures. The qualitative analyses were used to explore participants' learning processes and gaming strategies. The results indicated that the model well describes the problem-based gaming process in which the reflection phase seems to be a vital factor. Furthermore, the changes in playing strategies were found to enhance learning.
\end{abstract}

Keywords: educational game, game design, problembased learning, reflection, learning by teaching

\section{Introduction}

In recent years game studies have rapidly developed into an important research field. Naturally, the growth of game industry has aroused interest also among educational technology researchers. Unfortunately, it seems that educational games are seen as new magic tools that will solve the problems of computer-assisted learning. Educational games are argued to, for example, enhance learning, engage learners and provide such learning methods that correspond better with students' requirements and habits [1][2].

However, the real state of art of educational game research does not provide an account for such promises because research is mainly directed to the game design issues separated from learning. In fact, the examination of typical educational games indicates that games are primarily used as tools for supporting the practice of factual information in education ([3]). It can be argued that most educational games do not utilize the power of games as an interactive context-free media allowing players to actively test their hypotheses on phenomena and discovering new knowledge. The reason for this may be that the field of educational technology lacks research on how to design game environments that foster knowledge construction, deepen understanding and problem-solving [4][3] while being engaging and entertaining at the same time.

So far, mainly traditional educational models and theories have been used to explain learning with games [3]. Only a few attempts to integrate educational theories and game design aspects have been made [5][6][3]. However, most of these attempts have failed to achieve their primary goal - integration of the pedagogy and the game design aspects. Thus, more robust models that can be used as a foundation of educational game research are needed. The development of theoretical foundation for game-based learning is crucial because if educational games are developed without appropriate pedagogical basis, the investments in educational gaming technologies may be considered as wasted.

It is obvious that the research investments in educational games should be directed to develop a theoretical basis for game-based learning before the promises laid on educational games can be achieved. The aim of this paper is to evaluate the Problem-Based Gaming (PBG) model that has been recently proposed [7]. The PBG model is the latest and the most promising attempt to describe the learning mechanism in educational games. In this paper, the PBG model will be evaluated through a geometry game designed to support players' reflective thinking. 


\section{Problem-based Gaming Model}

In this section, the problem-based gaming model is shortly presented (more detailed description in [7]) in order to be able to ground the hypotheses to be studied (see Figure 1). The model describes learning as a cyclic process through direct experience $[8]$ in the game world. Learning is defined as a construction of cognitive structures through action in the game world. The model does not consider gaming either as individual or social activity, because games can consist of both individual and social events.

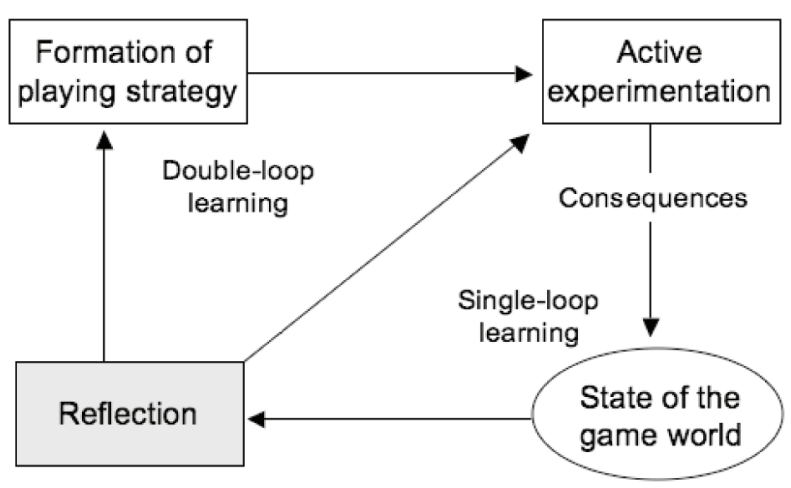

Figure 1. Problem-based gaming model.

When playing the game, a player tries to form appropriate playing strategies in order to solve the problems that the game provides to him/her. In the beginning of the game the player forms a playing strategy based on his/her prior experiences. After strategy formation, the player tests his/her strategy and possible hypotheses in the game world and observes the consequences of his/her actions. After the active experimentation phase, the player reflects his/her experiences about the utilized strategy. The feedback that the game provides from the player's actions should support reflective thinking by focusing his/her attention to relevant information from the learning point of view. The outcome of the reflection phase may be personal synthesis of knowledge, validation of hypothesis laid during the playing strategy formation, or a new strategy to be tested.

Reflection is a vital element in PBG process. However, according to [9] the phase of experiential learning in which people tend to be most deficient is reflection. On the grounds of the reflection a player decides whether he/she continues to apply the previously formed playing strategy or focuses his/her attention to changed variables of the game world in order to create better playing strategies. One-sided playing strategy refers to single-loop learning [10] which is neither effective nor learning developing method because it does not aspire to a better understanding of the problem domain. In contrast, the double-loop learning [10] emphasizes the scrutiny of the state of the game in order to generate better playing strategies and solutions to the problems. It is important that the player endeavours to test different kinds of strategies in order to expand knowledge on the subject matter.

\section{Method}

The main objective of this paper is to evaluate the structure of PBG model. How does the structure of the PBG model match with learning process in games? The hypotheses derived from the PBG model are following:

$\mathrm{H}$ 1. The changes of playing strategies increase the learning outcome.

H 2. Reflection stimulates double loop learning.

These hypotheses are formed only to reflect the main objectives of this research and they are not statistically tested in this study.

\subsection{Participants}

The experiment group consisted of 24 pre-school children ( 6 years old), 15 boys and 9 girls. The children were from three kindergartens nearest to the research site. They were not selected; all children from these kindergartens participated in this study.

Originally, there were 26 participants, but two children got sick on the post-test day and, therefore, there is no post-test result for them. Those two children are excluded from the analysis. 


\subsection{Materials}

The geometry game [11] utilized in this study relays on learning by doing [12] approach. In the game a player takes the role of a teacher. In the beginning of the game, the player meets her own virtual pet, an octopus, which wants to learn geometry. The task of the player is to put questions about geometrical shapes to her octopus and evaluate its answers. The tasks are 'what does not belong to the same group' type of questions as illustrated in Figure 2. The octopus answers according to its previous knowledge that is based on the tasks the player previously taught it. If the octopus does not know the answer to a certain question or if the question is badly designed, it will guess the answer.

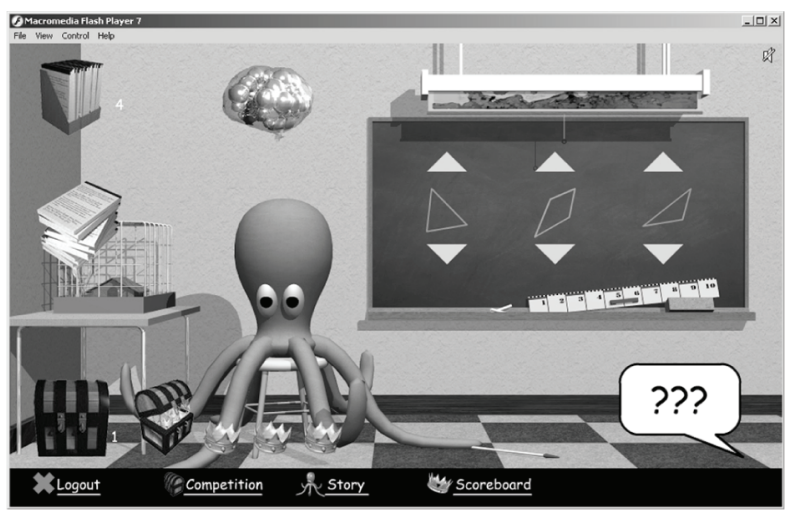

Figure 2. The teaching room.

The game AI is based on relations between geometrical concepts. Each teaching phase is recorded into a concept network, for example concept $\mathrm{A}$ belongs into the same group as the concept $\mathrm{B}$, or the concept $\mathrm{A}$ does not belong into the same group as the concept B. In the game, these relations were used logically: if the answer of the octopus was evaluated to be correct, two 'does not belong' relations and one 'belongs' relation were added to the octopus's concept structure. On the other hand, if the answer of the octopus was evaluated to be wrong, one 'does not belong' relation was added to the octopus's concept structure.

During the game, the conceptual structure of the octopus develops. When the octopus achieves a certain size of concept network, it will start to conclude. For example, if the octopus knows that the concepts A and B belong into the same group and the concepts $\mathrm{A}$ and $\mathrm{C}$ belong into the same group, it can conclude that $\mathrm{B}$ and $\mathrm{C}$ belong into the same group. On the other hand, if the octopus knows that the concepts $\mathrm{A}$ and $\mathrm{B}$ belong into the same group and the concepts $A$ and $\mathrm{C}$ belong into different groups, it can conclude that $\mathrm{B}$ and $\mathrm{C}$ belong into different groups.

An interesting element of teaching approach is the possibility to teach octopus wrongly. Naturally, a wrong teaching can be corrected by teaching the correct structure enough times. However, it takes time to override the wrong learning, because the game uses all taught information behind its decisions.

The geometry game does not restrict only to teaching the octopus, but a player can send his/her own octopus to compete against other octopuses. The other octopus (the opponent) has been taught by some other real life child, which gives a collaborative dimension to the game. The competition is based on the results of previous teaching, and that is directly reflected on the results. The competition situation is illustrated in Figure 3.

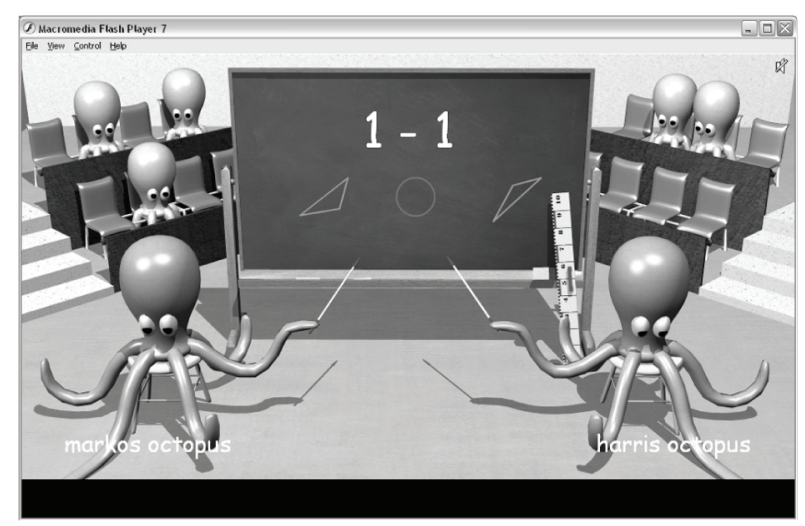

Figure 3. Competition in progress.

\subsection{Measures and Analyses}

This study is an empirical action study involving both pre- and post tests. The aim of the pre- and post tests was to estimate the learning outcome affected by the game. The test instrument contained tasks related to 1 ) recognizing basic geometric shapes, 2) analyzing the differences between the shapes and 3) producing basic geometric shapes. The control group was not needed to estimate the learning effect of the 
test instrument because the instrument was validated in previous studies [13] and the learning effect of the instrument was known (approximately $7.6 \%$ ).

The learning outcome was used as a grouping variable in this study and it cannot be transferred outside this context as a numeric result. Participants in the study were divided into two groups, according to the relative learning outcome. The individual relative learning outcome was defined as follows (1):

$$
(t 2-t 1-(\text { avg } t 2 \text { control }-t 1 \text { control })) / t 1
$$

where $\mathrm{t} 2$ is participant's score from the post-test, $\mathrm{t} 1$ is participant's score from the pre-test and (avg t2control-t1control) is the average learning outcome of the instrument, defined in previous studies. By taking the average learning outcome of the test instrument into account, the results were expected to be more realistic. The groups were defined as follows:

Gainers-group: Those who achieved more than $10 \%$ improvement during the game play.

Non-gainers-group: Those who achieved less than $10 \%$ improvement during the game play.

The experiment group was observed 1) by logging all human computer interactions with computer and 2) by researcher (manual notes). Player's teaching process was logged along with other HCI and it was possible to analyze this teaching process afterwards.

Because of the small sample size the results are analyzed by qualitative methods. In order to make the concept networks easier to understand and analyze, the concept networks were drawn as graphs, where each node represents a concept and vertices represent relations between the nodes. The graphs were visualized by cMapTools [15] concept mapping tool, even though the graphs were not correct concept maps. The reason to use cMapTools was the fact that conversion between gameAi and cMapTools was relatively easy because of cMapTool's XML import features. Concept maps used in the analysis of this paper are reported in [14].

\subsection{Procedure}

Before the playing session, all the children did the pre-test. During the pre-test they were in- structed and guided very formally. It took approximately 10 minutes to complete the pretest. Game play was started immediately after the pre-test.

The experiment group played the game in three subgroups. In every game session, there were two researchers and two kindergarten teachers present who helped the players if needed. They also discussed with them about gaming etc. A game play lasted approximately two hours. The children were allowed to talk with each other during the game and they were allowed short breaks for individual needs. In fact, they only took breaks for going to the toilet. All other needs were minor compared to the game play.

The post-test was done a day after the game play. Post-test was similar to the pre-test, as a test as well as a procedure.

\section{Results}

The results of the study are presented according to classification between gainers and nongainers.

\subsection{Gainers}

More than half of the players were classified to gainers-group $(n=14)$. The qualitative analysis of gainers' concept structures indicates that at the beginning of the game gainers had some unclear concepts in geometry. The players who benefited most from the game had several 'observed problem - reformulated strategy - corrected problem' patterns. In other words, during the gaming session they noticed their misconceptions and started to teach their octopus correctly.

Typical gainer's concept structure consisted of several concepts that were connected to other concepts by labels 1) belongs into the same group and 2) does not belong into the same group. The gainers taught their octopuses complex conceptual structures that covered the learning domain well. The corrected teaching behavior and corrected strategieswere the connective 
factor in gainer's group. However, the size of the conceptual structures did not predict learning.

Overall, the results indicate that the game supported reflective thinking in gainer's group. The main game elements that triggered reflection were animated competitions against other players' octopuses and the growth of octopuses' brains that was used to visualize learning in the game. On the basis of reflection, players could change their playing strategy to more effective one. This finding supports the structure of PBG model, emphasizing the role of reflection in strategy formation. The players who could evaluate the state of their octopus according to provided feedback tended to be doubleloop learners that facilitated learning. These results support the laid hypotheses.

\subsection{Non-gainers}

Approximately 40 percent of the players were classified into non-gainers-group. The conceptual structures varied a lot in this group. The size of the conceptual structure taught did not explain the relatively low learning outcomes in this group: Both the biggest and the smallest structure were found in this group. The pretest results indicated that the prior knowledge did not explain this variation. Furthermore, the total number of conceptual errors was found to be approximately as high in both gainers and non-gainers-groups.

The major difference between groups was in the amount of corrected errors: The non-gainers did not correct their misconceptions during the game play. The feedback channels, animated competitions against other players' octopuses and the growth of the octopuses' brains, did not stimulate reflective thinking in non-gainers. They did not either perceive the reasons that made their octopuses perform poorly in competitions or they did not understand the idea of the game. If the feedback that a game provides does not stimulate reflective thinking in players, the learning results are usually poor, as the results of non-gainers-group indicate.

Inadequate reflection can be also seen as poor gaming strategy formation, as the strategies of non-gainer's group indicate. Players could not develop adequate teaching strategies to achieve the goal of the game. Actually, several members of this group taught only 'are different group' relations. These players can be considered as single-loop learners supporting the laid hypotheses. Thus, it can be argued that reflective thinking is a vital element in game-based learning dividing players to single-loop and double-loop learners. The results also showed that double-loop learners tend to achieve better learning outcomes than single-loop learners. These findings are consistent with earlier results on this theme [7].

However, it is possible that poor gaming strategies and the resulting poor performance in the game may have confused some of these pupils leading to failure in the post-test. Thus, more research with larger sample sizes on the topic is needed in order to make more valid generalizations.

\section{Conclusions}

In this paper, the problem-based gaming model was studied through Geometry game. The results indicated that the model well describes the problem-based gaming process in which the reflection phase seems to be a vital factor. The outcome of the reflection phase may be personal synthesis of knowledge, validation of hypothesis laid or a new playing strategy to be tested. On the grounds of the reflection a player decides whether he/she continues to apply the previously formed playing strategy or focuses his/her attention to changed variables of the game world in order to create new playing strategies. It seems that reflection that stimulates double-loop learning [10] enhances learning, because players tend to aspire to a better understanding of the problem domain by actively testing different strategies.

The results indicate that the greatest challenge of the educational game design is to implement such game elements that trigger reflection during game play and support double-loop learning. Thus, the key issue in game design is to ensure that the game provides constructive and immediate feedback to the player. The meaning of such feedback is not emphasized in problembased gaming model. Therefore, the model should be complement with the gulf of evaluation [15] as proposed in Figure 4. 


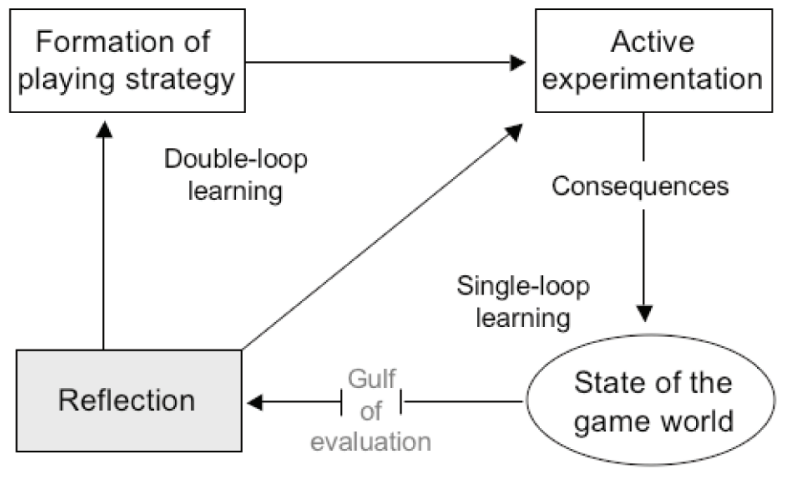

Figure 4. Complemented PBG Model.

The gulf of evaluation refers to a player's problems to perceive the consequences of his/her actions in the game. Inadequate perception of the consequences usually leads to failure of reflective thinking and may even lead to misconceptions.

As a summary, we can say that if there is something new to learn in the game, the effective learning game should encourage the learner to develop his/her mental conceptual structure or reformulate his/her gaming strategy. This can be achieved through games that support reflective thinking and provide possibilities to use different strategies.

\section{References}

[1] M. PREnSKY, Digital Game-based Learning. McGraw-Hill, New York, 2001.

[2] J. A. BETZ, Computer games: Increase learning in an interactive multidisciplinary environment. Journal of Educational Technology Systems, 24 (1995), 195-205.

[3] K. KIILI, Digital Game-based Learning: Towards an Experiential Gaming Model. The Internet and Higher Education, 8 (2005), 13-24.

[4] R. Moreno, R. E. Mayer, Role of guidance, reflection, and interactivity in an agent-based multimedia game. Journal of Educational Psychology, 97 (2005), 117-128.

[5] C. N. QuINN, Designing educational computer games. In: K. Beattie, C. McNaught, S. Wills, editors. Interactive multimedia in university education: Designing for change in teaching and learning, (1994) pp. 45-57. Elsevier, Amsterdam.

[6] A. Amory, R. SEAGRAM, Educational game models: Conceptualization and evaluation. South African Journal of Higher Education, 17 (2003), 206-217.
[7] K. KIILI, Foundation for Problem-Based Gaming. British Journal of Educational Technology, (2007), in Press.

[8] D. KolB, Experiential learning: experience as the source of learning and development. Prentice Hall, New Jersey, (1984).

[9] D. S. DuLEY, Field experience education. In: A. W. Chickering, editors. The modern American college, (1981) pp. 600-613. Jossey-Bass, San Francisco.

[10] C. ARgYris, D. SchÖN, Theory in practice: Increasing professional effectiveness. Jossey-Bass, San Francisco, 1974.

[11] H. Ketamo, M. Suominen,, (2005), AnimalClass. http://amc .pori.tut.fi/game/ [1/31/2007].

[12] J. DEWEY, Experience and Education. Simon and Schuster, New York, 1938/1997.

[13] H. Ketamo, An Adaptive Geometry Game for Handheld Devices. Educational Technology \& Society, 6 (2003), 83-95.

[14] H. Ketamo, M. SuOminen, AnimalClass - conceptual structures after game play. Research paper, (2006). http://amc.pori.tut.fi/game/ conceptual_structures.pdf [1/31/2007].

[15] D. NORMAN, The psychology of everyday things. Basic Books, New York, 1998.

Received: June, 2007 Accepted: September, 2007

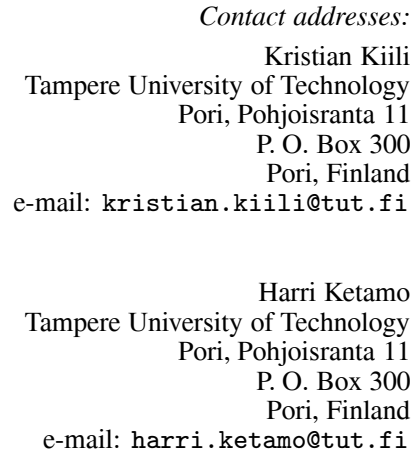

KRISTIAN KIILI works as a senior researcher at Tampere University of Technology. Currently, his research focuses on game-based learning, measurement of playing experience and educational game design.

HARRI KETAMO works as a senior researcher at Tampere University of Technology. His research focuses on user modeling, artificial inteligence and educational technologies. Currently, his work is funded by the Academy of Finland. 